\title{
LENGUAJE, INTERTEXTUALIDAD Y BIBLIOGRAFÍA EN LOS TRABAJOS DE INVESTIGADORES EN EDUCACIÓN EN CIENCIAS
}

\author{
MACÍAS, ASCENSIÓN y MATURANO, CARLA INÉS \\ Instituto de Investigaciones en Educación en las Ciencias Experimentales. \\ Facultad de Filosofía, Humanidades y Artes. Universidad Nacional de San Juan. \\ Av. José I. de la Roza, 230 oeste (5400) San Juan. Argentina.
}

\begin{abstract}
SUMMARY
The present work consists of an analytic revision of part of the existing literature on the teaching of Science in daily publications. The analysis was based on the articles published in Enseñanza de las Ciencias. Such analysis allows us to reflect on some of the devices used by the authors of magazine articles such as neologisms, quotes (intertextuality), bibliography and the way they use them in the text.
\end{abstract}

\section{INTRODUCCIÓN}

Las revistas de difusión de trabajos de investigación en educación en las ciencias son un material valioso que permite a investigadores y profesores acceder al pensamiento de quienes incursionan en este campo. También posibilitan conocer qué está sucediendo en innovación educativa y cómo son los movimientos tanto individuales como de grupos (Mata y Méndez, 1985). Dichas publicaciones son el vínculo entre la comunidad científica y profesional de un campo determinado (Jiménez Aleixandre y García-Rodeja, 1997); incluyen trabajos que corresponden a las investigaciones y reflexiones de un mundo particularmente importante para quienes lo han llevado a cabo (Sanmartí y Azcárate, 1997); y buscan estimular la investigación facilitando la comunicación por ser un medio de divulgación (Moreira, 1994).

A las reflexiones de los autores citados en el párrafo anterior que fueron publicadas en estos últimos años en
Enseñanza de las Ciencias, queremos sumar las nuestras referidas al análisis del lenguaje, la intertextualidad y la bibliografía que se utilizan en los artículos de la mencionada revista.

Los investigadores en educación en las ciencias y los docentes que se acercan a actualizarse en los nuevos aportes de las investigaciones sobre educación están acostumbrándose, en estos últimos años, a encontrarse que las publicaciones tienen nuevos vocablos. Éstos poseen un significado especial en este campo del saber. Muchos de ellos no están definidos en los diccionarios o las enciclopedias, o sus acepciones, desde el punto de vista de estos últimos, no se adaptan al contexto en que se utilizan en las publicaciones referidas.

A modo de ejemplo, quienes hemos seguido los aportes de la psicología de Ausubel encontramos nuevos térmi- 
nos como, por ejemplo, inclusor (Novak, 1985). En otros trabajos provenientes de traducciones hechas al español se utilizan las palabras subsumidor o subsunsor (Moreira, 1992) para referirse al mismo concepto. Éstas son usadas por dichos autores para explicar la teoría de la organización del conocimiento aun cuando no están incorporadas a nuestro lenguaje común.

De forma similar, encontramos el término molar en diferentes publicaciones de psicología. Tal es el caso de Sierra y Carretero (1993), que definen los esquemas como «estructuras y procesos mentales insconscientes que subyacen a los aspectos molares del conocimiento y de las destrezas humanas (p. 145)». Según el diccionario de la Real Academia Española (1995), molar significa «perteneciente o relativo a la muela» o «apto para moler», acepción que debe adaptar el lector a fin de interpretar el texto citado.

Sabemos que docente es la persona que enseña, el profesional de la enseñanza que, según el Diccionario Enciclopédico Salvat (1986), ejerce esta acción en posesión de un título académico. En estos últimos tiempos y como producto de los nuevos aportes que la reforma educativa ha producido se lo ha llamado capacitador. El significado de este concepto se limita a considerar capacitador a una persona apta o habilitada para realizar algo. Vemos que el uso de este vocablo delimita lo que es la acción del docente cuya labor es mucho más abarcativa.

La utilización de vocablos, acepciones o giros nuevos realiza aportes a nuestro idioma. Como consecuencia, nos planteamos algunas preguntas, cuestionándonos sobre ciertas situaciones que consideramos conveniente analizar. Si las personas que se acercan a las publicaciones no están familiarizadas con este lenguaje, ¿cómo pueden entender lo que el autor quiere expresar si se presentan vocablos a veces incomprensibles? Es importante destacar que el lenguaje es una propiedad social y que, además de preservarla, debemos mejorarla. ¿Hasta qué punto debe aceptarse la incorporación de todos esos términos que a diario nos llegan? ¿Hasta qué punto debemos convertir nuestros trabajos en una colección de palabras «nuevas»? ¿Hasta qué punto es responsabilidad de los autores aclarar la acepción con que se usan las palabras cuando éstas difieren del significado corriente?

Por otra parte, observamos que los autores de artículos mencionan en sus trabajos a otros autores, libros o publicaciones (intertextualidad). A veces, no sólo hacen mención sino que citan textualmente a otros, reproduciendo exactamente un texto. Destacamos la importancia de estos recursos, ya que sitúan al lector en el marco referencial del trabajo. El detalle de las fuentes genera la bibliografía. Cabe plantearnos si existe un punto de equilibrio entre la falta del uso de estos recursos (lo que podría significar una gran originalidad o una total omisión de los referentes) y el exceso (que implicaría para el lector, tal vez, una exuberancia en lo que se refiere a la información sobre autores y fechas, lo cual puede dificultar la comprensión de lo que se lee).
Sobre la base de estas reflexiones nos proponemos realizar una revisión analítica de una parte de la literatura existente sobre la enseñanza de las ciencias, específicamente en la revista Enseñanza de las Ciencias desde el año 1990 hasta la fecha. No sólo nos dedicaremos, en este trabajo, al análisis del lenguaje utilizado, sino también al de aquellos recursos usados por los autores de los artículos como son los neologismos y las citas (intertextualidad), entre otros, y el manejo que se hace de éstos en el texto.

\section{LENGUAJE,INTERTEXTUALIDADY BIBLIO- GRAFIA EN LAS PUBLICACIONES}

En nuestra actividad consultamos a menudo, como lo hemos manifestado, las publicaciones periódicas, dado que constituyen una referencia obligada para la investigación educativa. Hemos notado que las exigencias para el envío de trabajos a las revistas o reuniones científicas de habla castellana, pasan por el tipo de trabajo (original) y la forma de presentación (número de copias, cantidad de líneas del resumen, disco flexible con el tipo de procesador, etc.), pero no conocemos la existencia de una referencia explícita en lo que respecta a la gramática.

El lenguaje que usan los investigadores tiene términos que provienen del lenguaje ordinario en opinión de Klimovsky (1995). Según este autor, el vocabulario puede aportar cuestiones de vaguedad y puede presentar el fenómeno de pluralidad de significaciones o polisemia. Aconseja iniciar los trabajos rescatando significados y precisando definiciones. Consideramos que esto redundaría en beneficio de la comunidad a la que están dirigidos dichos trabajos.

Apreciamos, en forma general, que en las mencionadas publicaciones es cada vez mayor el uso que se hace de ciertos recursos en el lenguaje escrito como son los que se exponen a continuación.

\section{Utilización de neologismos}

En el estudio del lenguaje se considera que las nuevas palabras con significado que se incorporan en el uso diario, o como acepciones que utilizan ciertos colectivos con intereses comunes, son las que nutren y enriquecen el idioma. Las mismas pueden surgir de diferentes modos: derivación, composición u otras variaciones de palabras existentes, origen en otra lengua, invención, etc. Estos vocablos se denominan neologismos. Así, por ejemplo, la utilización de nuevas palabras y su inclusión puede deberse a que algunos vocablos nuevos que se originan en otra lengua no tienen una traducción directa al castellano; es decir, que no existe una palabra en nuestro lenguaje que indique exactamente la misma idea o concepto al que hace referencia la palabra original. En ese caso se usa la palabra en el idioma original o se la deforma «castellanizándola», dando lugar a un neologismo. Tal es el caso de los términos relacionados con la 
informática. En otros casos, dichas palabras tienen su traducción a nuestra lengua y, sin embargo, se usan en el otro idioma. Sea cual fuere la forma en que se originen, se convierten en material enriquecedor, dado que son el fruto de la incorporación de palabras derivadas de otras lenguas o de nuestro propio idioma que todavía no figuran en el diccionario, pero que se usan en ciertos ámbitos.

\section{Intertextualidad}

Eco (1988) considera la intertextualidad como textos que citan otros textos, siendo el conocimiento de los textos precedentes una condición necesaria para entender lo que se lee, lo cual es un procedimiento típico de la literatura postmoderna. Este recurso también se utiliza en películas, obras de teatro y en la elaboración de material informativo tanto para televisión como para otros medios.

Lemke (1997) expresa lo siguiente:

«Cuando participamos en una actividad como leer un texto o comprender el habla u otras formas de acción social significativa, relacionamos palabras o eventos con patrones familiares. Puede tratarse de palabras y eventos en el mismo texto o secuencia de acción, o de palabras y eventos de distintos textos o tiempos. Éste es el principio de la llamada intertextualidad...» (p. 216).

Para este autor, los recursos intertextuales son textos que comparten el mismo patrón (textos cotemáticos), o de la misma estructura de actividad (textos coactivos), o bien del mismo tipo de género (textos cogenéricos). Además nos aporta las siguientes consideraciones:

«Las prácticas de conexión de una comunidad son una parte importante de las formas de elaborar significados. Podemos elaborar significados a través de la relación entre dos textos que no se pueden elaborar por medio de un solo texto...» (p. 217).

Consideramos que los recursos intertextuales permiten establecer relaciones entre el autor del texto, los autores citados y el lector. Los aportes resultantes serán interesantes a partir de las propias interpretaciones que haga este último.

Por otra parte, las fuentes consultadas en cada trabajo tienen su reseña bibliográfica o referencias.

\section{Bibliografía}

La bibliografía consiste en el catálogo de los textos citados en los artículos. La misma incluye el o los autores, año de edición, editorial y lugar de publicación. La bibliografía sitúa al lector en las fuentes teóricas y empíricas en que se basa el autor (Jiménez Aleixandre y García-Rodeja, 1997).

\section{DISEÑO DEL ANÁLISIS. SELECCIÓN DE LA MUESTRA}

Con el fin de hacer un estudio para profundizar el análisis surgido de nuestras inquietudes, hemos seleccionado la revista Enseñanza de las Ciencias. La elección se fundamenta en que es una de las publicaciones periódicas que consultamos con frecuencia en nuestra actividad como docentes e investigadores. Por otra parte, coincidimos con las directoras del consejo de redacción de la misma (1994) que en la carta-editorial expresan que «es una referencia obligada para la investigación en didáctica de las ciencias y de las matemáticas, particularmente en nuestro país y en el ámbito iberoamericano» (p. 1).

Por el tipo de análisis que pretendemos hacer, hemos seleccionado aleatoriamente tres artículos de la revista por año, a partir de 1990, siguiendo la misma forma de selección al azar para cada año, salvo en 1997, que tomamos solamente dos. De esta manera, la muestra está constituida por 23 artículos que fueron examinados exhaustivamente. Los mismos serán identificados en adelante indicando el número de artículo (1, 2 ó 3) seguido del último número del año de publicación; por ejemplo, el artículo 25 es el segundo artículo analizado del año 1995. Hemos procedido así para no indicar expresamente los trabajos de la muestra ni la línea y el campo de investigación.

Pautamos el análisis teniendo en cuenta los siguientes criterios de selección:

\section{1) Utilización de neologismos}

Hemos marcado en cada artículo las palabras que no figuran en el diccionario. Para esto hemos constatado en el Diccionario de la Lengua Española aprobado por la Real Academia Española (1992) y en el Diccionario Enciclopédico Salvat (1986) para asegurarnos de que fueran neologismos.

\section{2) Intertextualidad}

Para este caso hemos fijado como criterios los siguientes:

Menciones. Se refiere al recurso utilizado por algunos autores que mencionan a investigadores o científicos cuyas teorías se suponen conocidas por los lectores del artículo. Por ejemplo, es el caso de Ausubel, Darwin, Piaget, Novak, Kuhn, etc. En estos casos no hay referencia a una obra específica del investigador sino que versa sobre alguna idea consensuada a partir de su teoría o de sus estudios.

Menciones de autores. Analizamos en los artículos la inclusión de menciones de autores cuyas obras figuran en las referencias bibliográficas. En las mismas, además del apellido, se indica el año que corresponde a la publicaciones. Por ejemplo: Novak, 1985.

Menciones de publicaciones. Bajo este ítem hemos englobado aquellas menciones de publicaciones que se 
hacen de forma expresa, como, por ejemplo: manuales, libros o revistas (obviando al autor/es). Por ejemplo, el Diccionario de la Real Academia Española (1992).

Citas textuales. Nos referimos a las transcripciones de frases tomadas textualmente de una obra del autor citado, que se acostumbran a poner entre comillas $\mathrm{y}$, en algunos, casos con letra cursiva.

Otras citas textuales. Son aquellas citas textuales sacadas de encuestas, pruebas tomadas a alumnos, entrevistas, etc. La fuente no se incluye en la bibliografía y es, por lo general, parte del trabajo que se presenta en el artículo.

\section{Referencias bibliográficas}

Con el objeto de analizar la bibliografía mencionada por los autores que está incluida al fínalizar cada artículo, hemos clasificado cada publicación según el siguiente criterio:

- Libros

- Revistas

- Otras publicaciones

Cabe que aclaremos que en el último ítem hemos incluido: documentos presentados en congresos y publicados en las actas o resúmenes; tesis doctorales o de maestría publicadas o sin publicar; publicaciones internas de departamentos o institutos de universidades $u$ otros organismos tales como informes finales de proyectos o documentos de entidades como UNESCO, OEA, etc. y trabajos no publicados.

\section{ANÁLISIS DE LOS RESULTADOS}

Ahora nos dedicaremos a hacer algunos comentarios referidos a los artículos de Enseñanza de las Ciencias analizados. Los mismos están agrupados en los tres ítems según detallamos anteriormente.

\section{Utilización de neologismos}

En algunos artículos aparecen palabras que no figuran en el diccionario y enciclopedia consultados. Tal es el caso de los siguientes términos: hipotetizar (que pudo expresarse como enunciar una hipótesis) y constructo (está utilizado dentro de la teoría de Ausubel como una construcción del conocimiento). También encontramos holística, inclusor, conductista, entre otros. En algunos artículos aparecen palabras en otros idiomas, especialmente en latín o inglés. Por ejemplo: radiocasete, theories, homo sapiens, pull-down, software, problem-solving, continuum, entre otras. Cabe que señalemos que no se observan, en general, muchos neologismos. Nos preguntamos si los asesores de los trabajos tienen especial atención para que no proliferen los mismos.

\section{Intertextualidad}

Menciones, menciones de autores y de publicaciones

Se observa, en general, que las menciones de investigadores supuestamente conocidos por la mayoría fluctúan entre 0 y 25 por artículo de la muestra. El valor medio obtenido es 5 menciones por artículo.

Algunos de los nombres más mencionados son los que figuran en la tabla I.

Tabla I

Menciones de autores en los artículos.

\begin{tabular}{|l|c|}
\hline $\begin{array}{l}\text { Investigador } \\
\text { mencionado }\end{array}$ & $\begin{array}{c}\text { Cantidad de veces } \\
\text { que se menciona } \\
\text { (expresado en porcentaje } \\
\text { de la muestra) (\%) }\end{array}$ \\
\hline Novak & 12 \\
\hline Ausubel & 10 \\
\hline Darwin & 5 \\
\hline Lamarck & 4 \\
\hline Ampere & 3 \\
\hline Piaget & 3 \\
\hline Moreira & 3 \\
\hline $\begin{array}{l}\text { Otros (Pasteur, Koch, } \\
\text { Jiménez-Aleixandre,Oersted, } \\
\text { Popper, Avogadro, etc.) }\end{array}$ \\
\hline
\end{tabular}

En lo que respecta a las menciones de autores, observamos que en los trabajos se hace referencia a otro autor indicando, de forma explícita, el año de la obra correspondiente, la cual forma parte de la bibliografía. Se realiza con mayor frecuencia que en el caso anterior. Los valores obtenidos en este análisis fluctúan entre 8 y 103 , siendo solamente tres de las publicaciones analizadas las que superan las 80 menciones de autores. El valor medio obtenido para la muestra es 34 menciones por artículo. Resulta interesante destacar que, al contabilizar los casos en que los autores se mencionan ellos mismos, encontramos que, en 16 artículos de la muestra, los autores hacen referencia hasta 13 veces a sus trabajos anteriores.

Respecto a las menciones de publicaciones, notamos que muchos artículos incluyen menciones de una misma publicación en varias oportunidades. En uno de ellos se cita seis veces la misma publicación de un autor. Con respecto a la mención directa de la publicación a la que se hace referencia, obviando el nombre del autor, los valores obtenidos oscilan entre 0 y 18 , tomando un valor medio de 3 menciones por artículo. Solamente en 15 de los 23 trabajos analizados se utiliza este recurso. Por otra parte, hemos observado que, en algunos casos, se citan publicaciones que no se incluyen en las referencias bibliográficas. 
En el gráfico 1 podemos observar la cantidad de menciones, menciones de autores y de otras publicaciones para cada artículo. De forma separada están indicadas con diversos trazos.

Gráfico 1

Menciones, menciones de autores y de publicaciones en los artículos de la muestra.

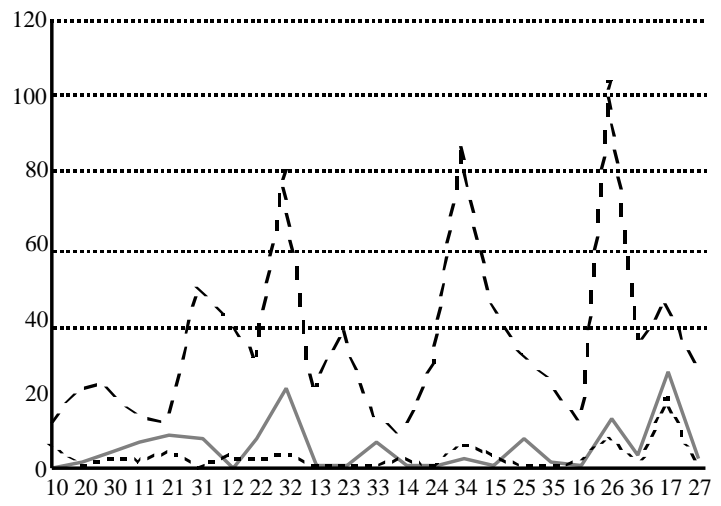

- - - menciones - - menciones de autores _ menciones de o.p.

Hemos observado que hay una tendencia a aumentar las menciones de autores en el transcurso del tiempo, como lo muestra el gráfico 2. En el mismo hacemos la recta de mejor ajuste cuya pendiente es positiva, lo que justifica la afirmación anterior.

Gráfico 2

Recta de mejor ajuste para las menciones de autores.

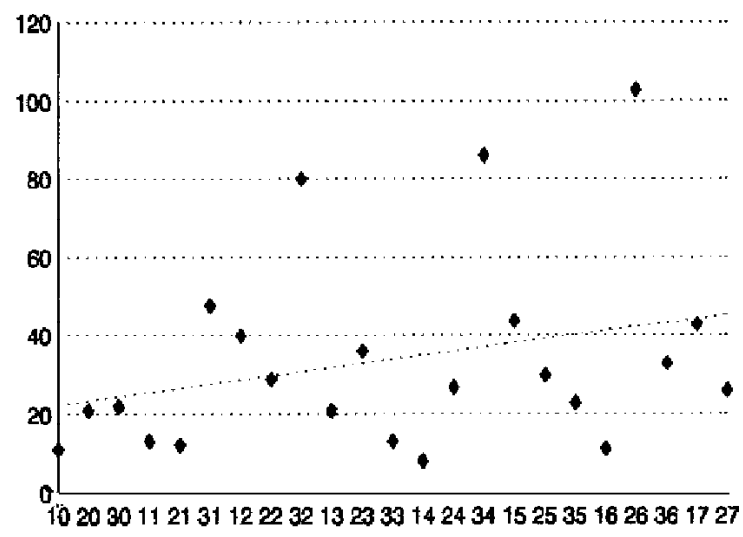

- menciones de autores

\section{Citas textuales}

Los valores obtenidos para las citas textuales de publicaciones mencionadas en la bibliografía oscilan entre 0 y 11 por artículo, tomando un valor medio de 3 . Hay 7 artículos que no hacen ninguna cita textual. En el gráfico 3 representamos la cantidad de citas textuales en función de cada uno de los artículos analizados y la recta correspondiente al valor medio.

Gráfico 3

Citas textuales en los artículos de la muestra.

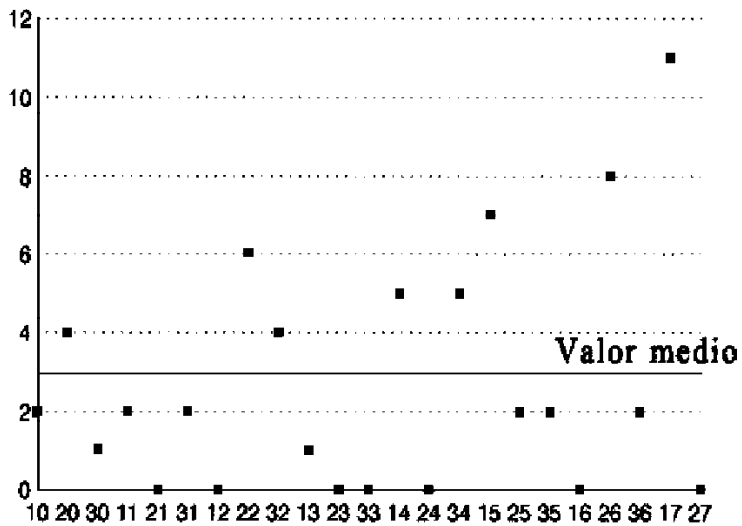

- citas textuales

Con referencia a lo que hemos llamado otras citas textuales, los resultados indican valores entre 0 y 23 . El valor medio obtenido es 2 y solamente en 5 de los artículos analizados se usa este recurso.

\section{Bibliografía}

En el gráfico 4 puede verse la cantidad de libros, revistas y otras publicaciones, por separado y para cada uno de los artículos analizados. La mayoría son revistas (62\%), siguen los libros (23\%) y por último las otras publicaciones $(15 \%)$. Los porcentajes están expresados sobre el total de los artículos.

Hemos contabilizado la mención de 381 revistas en las referencias de los 23 artículos, de las cuales 86 corresponden a la revista Enseñanza de las Ciencias, lo que representa un $23 \%$ del total.

Si promediamos para cada año la cantidad total de publicaciones mencionadas en la bibliografía, obtenemos el gráfico 5. En el mismo se ha incluido la recta de mejor ajuste cuya pendiente toma un valor positivo. Esto indica que el número de referencias bibliográficas por artículo tiene tendencia a incrementarse en los últimos años. 
Gráfico 4

Análisis de la bibliografía distinguiendo libros, revistas y otras publicaciones.

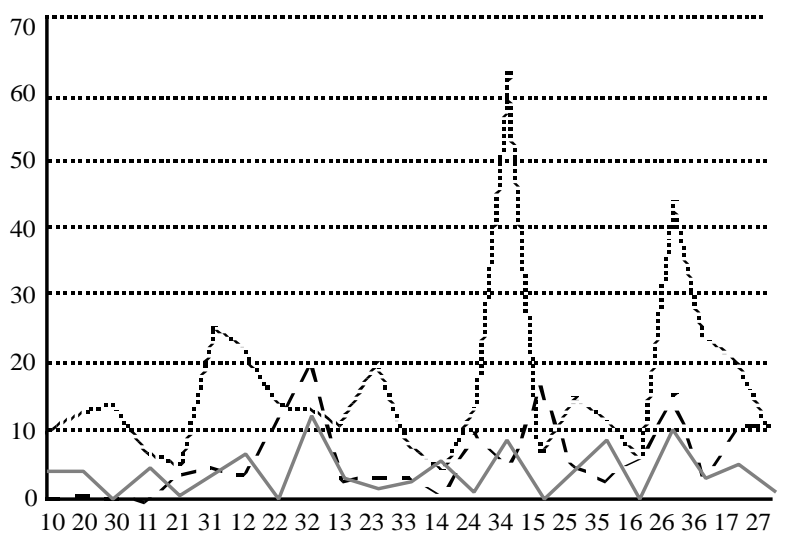

- libros $\ldots$. revistas $\quad$ - o.publicaciones

Gráfico 5

Total de publicaciones mencionadas en la bibliografía para los artículos de la muestra.

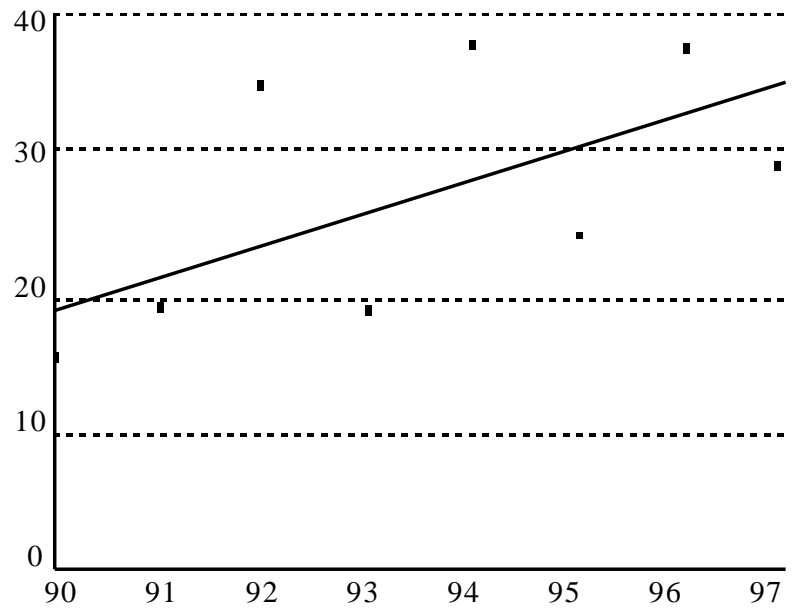

\section{REFLEXIONES}

En este artículo hemos querido explicitar algunas inquietudes que nos han ido surgiendo a lo largo de estos últimos años en que hemos recurrido con frecuencia a Enseñanza de las Ciencias. El análisis realizado muestra una vista parcial de algunos aspectos que consideramos de relevancia a la vez que se suma a las reflexiones de Moreira (1994), Sanmartí y Azcárate (1997) y Jiménez Aleixandre y García-Rodeja (1997).
De acuerdo con nuestro análisis, hemos observado que no se hace uso de muchos neologismos en los artículos de la revista Enseñanza de las Ciencias. Hemos notado que los autores más conservadores limitan el uso de esos nuevos términos» y utilizan los vocablos que significativamente mantienen el concepto y que sustentan el contexto.

No dudamos de que los nuevos aportes permiten que el lenguaje se mantenga vivo por acción de la incorporación de nuevas palabras. Pero, ¿hasta que punto es aconsejado? A veces se utilizan los neologismos sin necesidad, ya que nuestro idioma es rico en vocablos aceptados. Pensamos que las publicaciones son comunicaciones que deben ser claras y el uso de las palabras debe permitir que lo que se expresa sea concreto y preciso. Aunque las revistas de habla castellana que hemos consultado no exigen condiciones referidas al lenguaje para las publicaciones, creemos que este aspecto debería ser tenido en cuenta. Si el autor de un trabajo incluye un neologismo tendría la responsabilidad de definirlo de alguna manera.

Queremos destacar que no somos especialistas en lingüística pero que nos preocupamos por el uso correcto del lenguaje en la exposición de nuestros trabajos y en las lecturas que hacemos nos cautivan los textos claros, con rigor científico, lo suficientemente concisos para despertar nuestra imaginación y lo suficientemente extensos sin caer en exageraciones que no nos «muevan» a reflexiones.

En lo que se refiere a la intertextualidad presente en todos los trabajos consideramos que es un recurso típico, como hemos dicho, de la literatura postmoderna. Aquí caben ciertas reflexiones que surgen de preguntarnos si la proliferación de citas influye en la comprensión del texto. Citar entre comillas puede llevar al lector a no prestar atención al contenido de las citas sino a la forma de hacer el enlace con el nuevo texto, tal como lo expresa Eco (1988). Sin embargo, si esto no se hiciera parecería que las palabras citadas fueran propias del autor que cita y no del citado.

Las menciones de investigadores sin referencia a sus obras evidencian que el autor del artículo supone que son conocidos por el lector. Esto, en algunos casos, ocurre debido a que el autor domina cierto campo de conocimiento y juzga superfluo dar detalles sobre investigadores que avalan sus afirmaciones, olvidando que puede haber lectores que no los conozcan o no identifiquen aquella parte de su teoría a la que se hace referencia. A esto se agrega que estos últimos no puedan ampliar lo expuesto por no saber a qué fuente recurrir.

Las menciones de autores (cuya obra aparece en la bibliografía) y de publicaciones indican que los autores han querido destacar de la literatura anterior existente sobre el tema y los sitúan en un marco teórico determinado. La calidad de las menciones es uno de los criterios que son tenidos en cuenta por los evaluadores para medir la calidad de los trabajos (Jiménez Aleixandre y GarcíaRodeja, 1997), a lo que cabe agregar que deben tener 
especificidad (Sanmartí y Azcárate, 1997). En la utilización de este recurso puede caerse en excesos que son desaconsejados. Hay autores, como Carretero (1993), por ejemplo, que expresa, en uno de sus libros, que prefiere evitar la proliferación de citas bibliográficas, hecho común en los trabajos de carácter monográfico o académico, para facilitar al lector una mejor comprensión.

Bisquerra y Forner (1992) aconsejan no abusar de las citas textuales. Además, cuando se hacen citas textuales se saca una frase o párrafo fuera del contexto donde fue escrito para situarlo en otro. El riesgo que se corre con este procedimiento es que puede estarse cambiando el sentido con que la idea fue escrita originalmente. Creemos que el excesivo uso de este recurso no implica una mejor valoración del escrito. Consideramos que el número de citas no está en relación directa con el conocimiento y acceso a las fuentes de información que tiene el autor, por lo que, a nuestro criterio, debe evitarse en las publicaciones la proliferación de muchas citas y menciones de autores e investigadores, citas fuera de contexto o que no explicitan las ideas de forma concreta y, en consecuencia, no motivan reflexión alguna.

Con respecto a la bibliografía de los artículos analizados, hemos señalado que el número de revistas supera

\section{REFERENCIAS BIBLIOGRÁFICAS}

BISQUERA, R. y FORNER, A. (1992). Normativa sobre referencias bibliográficas y citas en el redactado. Revista Investigación Educativa, 19, pp. 81-87.

CARRETERO, M. (1993). Constructivismo y educación. Buenos Aires: Aique.

DICCIONARIODELALENGUAESPAÑOLA (1995). Madrid: Espasa-Calpe, SA.

DICCIONARIO ENCICLOPÉDICO SALVAT. (1986). Barcelona: Salvat Editores, SA.

ECO, H. (1988). De los espejos y otros ensayos. Barcelona: Lumen.

EDITORIAL DE ENSEÑANZA DE LAS CIENCIAS. (1994). Enseñanza de las Ciencias, 12(2).

ENSEÑANZA DE LAS CIENCIAS, 1990 a 1997.

JIMÉNEZ ALEIXANDRE, M.P. y GARCÍA-RODEJA GAYOSO, I. (1997). Hipótesis, citas, resultados: reflexiones sobre la comunicación científica en didáctica de ciencias. Enseñanza de las Ciencias, 15(1), pp. 11-19.

KLIMOVSKY, G. (1995). Las desventuras del conocimiento científico, Una introducción a la epistemología. Buenos Aires: AZ editora. ampliamente el de libros. Esto nos indicaría que la revista es un medio más actualizado de información. El libro, por otra parte, tarda más tiempo en llegar a los lectores y puede que resulte más difícil su adquisición.

Apuntamos a destacar que, en los artículos, lo importante es que deben ser comprensibles aunque estamos seguros de que ningún autor pretende que su obra sea poco clara y busca siempre que guste. Los trabajos publicados en el área de la enseñanza de las ciencias deben adaptarse tanto a los lectores expertos como a novatos. Debe servir a aquéllos que buscan en la lectura cotejar las ideas del autor con las propias y, en algunos casos, encontrar o enriquecerse con nuevos enfoques. También deben ser de utilidad para quienes desean conocer y reflexionar acerca de la investigación educativa para mejorar su acción en el aula o para encarar una investigación. Al escribir, el investigador debe tener en cuenta todos estos aspectos y procurar comunicar sin tecnicismos su trabajo usando un lenguaje claro, coherencia en las menciones con citas debidamente justificadas y bibliografía detallada que permita al lector acercarse a las fuentes pertinentes. Debemos considerar que no somos una individualidad. No escribimos para nosotros, sino para dar a conocer nuestras ideas al colectivo. Las publicaciones son, realmente, el medio de crear y recrear lo que la comunidad de investigadores en educación piensa.
LEMKE, J.L. (1997). Aprender a hablar ciencia. Lenguaje, aprendizaje y valores. Barcelona: Paidós.

MATA, A. y MÉNDEZ, A. (1985). La renovación didáctica de las ciencias experimentales. Estudio bibliométrico. Enseñanza de las Ciencias, pp. 3-10.

MOREIRA, M.A. (1992). Aprendizaje significativo, conocimiento científico y cambio conceptual. Conferencia dictada en la V Reunión Latinoamericana sobre Educación en Física. Porto Alegre. Brasil.

MOREIRA, M.A. (1994). Diez años de la revista Enseñanza de las Ciencias: de una ilusión a una realidad. Enseñanza de las Ciencias, 12(2), pp. 147-153.

NOVAK, J.D. (1985). Teoría y práctica de la educación. Madrid: Alianza Editorial.

SANMARTÍ, N. y AZCÁRATE, C. (1997). Reflexiones en torno a la línea editorial de la revista Enseñanza de las Ciencias. Enseñanza de las Ciencias, 15(1), pp. 3-9.

SIERRA, B. y CARRETERO, M. (1993). Aprendizaje, memoria y procesamiento de la información: la psicología cognitiva de la instrucción, en Coll, C., Palacios, J. y Marchesi, A. (comp.). Desarrollo psicológico y educación II. Madrid: Alianza Editorial.

[Artículo recibido en diciembre de 1997 y aceptado en septiembre de 1998.] 